\title{
Decision-Making in Complicated Geometrical Problems
}

\author{
Amir Mosavi \\ University of Debrecen \\ Egyetem ter 1. Debrecen, \\ Hungary
}

\begin{abstract}
Due to increasing the number of decision-making criteria in today's ever complicated geometrical optimization problems, the traditional multiobjective optimization approaches, whether a priori, a posteriori or interactive's, found to be insufficient and ineffective. In this paper the drawbacks of the current algorithms are reviewed and the urgent need for inserting a learning component in the optimization loop is discussed. In the following the methodology of reactive optimization for evolutionary interactive multiobjective optimization for solving complicated geometrical decisionmaking problems is adopted. The proposed brain-computer optimization follows to the paradigm of learning while optimizing, through the use of online machine learning techniques as an integral part of a self-tuning optimization scheme. At the end the effectiveness of the approach to geometrical problems is emphasized by providing the study case of optimal design problem of curves and surfaces.
\end{abstract}

\section{Keywords}

Decision-making, geometry, optimization

\section{INTRODUCTION}

We According to [3] the general form of the Multiobjective optimization (MOO) problems is stated as

Minimize $\mathbf{f}(\mathbf{x})=\left\{f_{\mathbf{1}}(\mathbf{x}), \ldots, f_{\boldsymbol{m}}(\mathbf{x})\right\}$ Subjected to $\mathbf{x} \in$ $\Omega$ where $\mathbf{x} \in \mathbb{R}^{n}$ is a vector of $n$ decision variables; $\mathbf{x} \subset \mathbb{R}^{\mathrm{n}}$ is the feasible region and is specified as a set of constraints on the decision variables; $\mathbf{f}: \Omega \rightarrow \mathbb{R}^{m}$ is made of $m$ objective functions subjected to be minimization. Objective vectors are images of decision vectors written as $\mathbf{z}=\mathbf{f}(\mathbf{x})=$ $\left\{f_{1}(\mathbf{x}), \ldots, f_{m}(\mathbf{x})\right\}$. An objective vector is considered optimal if none of its components can be improved without worsening at least one of the others. An objective vector $\mathbf{z}$ is said to dominatez', denoted as $\mathbf{z} \prec \mathbf{z}^{\prime}$, if $z_{k} \leq z_{k}^{\prime}$ for all $k$ and there exist at least one $h$ that $z_{h} \leq z_{h}^{\prime}$. A point $\hat{\mathbf{x}}$ is Pareto optimal if there is no other $\mathbf{x} \in \Omega$ such that $\mathbf{f}(\mathbf{x})$ dominates $\mathbf{f}(\hat{\mathbf{x}})$. The set of Pareto optimal points is called Pareto set (PS). The corresponding set of Pareto optimal objective vectors is called Pareto front $(\mathrm{PF})$

Solving a MOO problem would be done by providing the decision-maker (DM) with the optimal solution according to some certain utility criteria allowing to choose among competing PF. Such utility criteria are often inconsistent, difficult to formalize and subjected to revision.

Approaches to MOO are divided into the two broad categories of non-interactive and interactive ones [13]. According to [14], through interactive MOO the DM is building a conviction of what is possible and confronting this knowledge with the preferences that also evolve where learning task and the computer-supported solution processes are involved.

On the other hand the non-interactive approaches are divided into a priori approaches, where the preferences are formulated in advance, and a posteriori approaches, where a PS is created. A priori methods have the drawback of requiring the user to accurately pre-specify the preferences which is actually hard for a DM. A posteriori methods, on the other hand, imply a confusing selection task among a large and complicated set of candidate solutions.

\section{FROM EVOLUTIONARY ALGORITHMS TO BRAIN-COMPUTER OPTIMIZATION}

All Evolutionary algorithms (EAs) are among the most popular a posteriori methods to generating PS to a MOO problem. The evolutionary algorithms of MOO for solving MCDM problems have been around for almost twenty years now [15]. EA are ideally suited to search for a set of PS to be presented to the DM. In this paradigm, evolutionary multiobjective optimization algorithms (EMOAs) aim at building a set of points near the PF. Currently, most evolutionary EMOAs apply Pareto-based ranking schemes. Some of the most successful EMOAs [16] rely on Pareto dominance classification as a fitness measure to guide selection of the new population. The work [17] indicates that resorting to Pareto dominance classification to assign fitness becomes ineffective for increasing number of objectives and proposes a refined preference ordering based on the notion of order of efficiency [18].

MOO of curve and surfaces [5] would be a good example for such ineffective attempt and increasing complexity. The reviewed and applied approaches for solving the MOO of the curve and surfaces [5] whether a priori or a posteriori, in particular EA, involve plenty of various complications. The reason is that the proportion of $\mathrm{PF}$ in a set grows very rapidly with the dimension $\boldsymbol{m}$. In fact the reality of applied DM has to consider plenty of priorities and drawbacks to both interactive and non-interactive approaches. Although the mathematical representative set of the DM model is often created however presenting a human DM with numerous representative solutions on a multi-dimensional PF is very far from reality. This is because the typical DM cannot deal with more than a very limited number of information items at a time [19]. Therefore a satisficing decision procedures should be developed according to human memory and data processing capabilities.

Moreover often DMs cannot formulate their objectives and preferences at the beginning. Instead they would rather learn on the job. This is already recognized in the MOO formulation, where a combination of the individual objectives 
into a single preference function is not executed. Considering [5] the DM is not clear about the preference function. This uncertainty is even more increased when the objectives such as beauty involved. In fact the assumption that a fixed, deterministic and error-free preference structure of the DM is available is often not realistic. Imprecisions, contradictions, changes of judgment over time are the characteristics of most human decision processes. This fact would employ lots of uncertainty and inconsistency.

Interactive approaches try to overcome some of these difficulties by keeping the user in the loop of the optimization process and progressively focusing on the most relevant areas of the PF directed by DM. This is done when the fitness function is replaced by a human user. However most DM are typically more confident in judging and comparing than in explaining. They would rather answer simple questions and qualitative judgments to quantitative evaluations. In fact the number of questions that have to be asked from the DM before a satisfactory solution is identified is a crucial performance indicator of interactive methods. This would demand for selecting appropriate questions, for extracting as much information as possible from the answers, for building approximated models which may reduce the need to bother the DM.

The above priorities, as it is indicated in [20], demand a shift of paradigm, from building a set of solutions which is representative of the true $\mathrm{PF}$, to the interactive construction of a sequence of solutions, so called brain-computer optimization, where the DM is a crucial learning component in the optimization loop, a component characterized by limited rationality and advanced question-answering capabilities. This would be the motivation for the systematic use of machine learning techniques for online learning schemes in optimization processes. In fact in particular, the objective of reactive optimization (RO) [1] is to design problem solving schemes with an internal online feedback loop for the self-tuning of critical parameters.

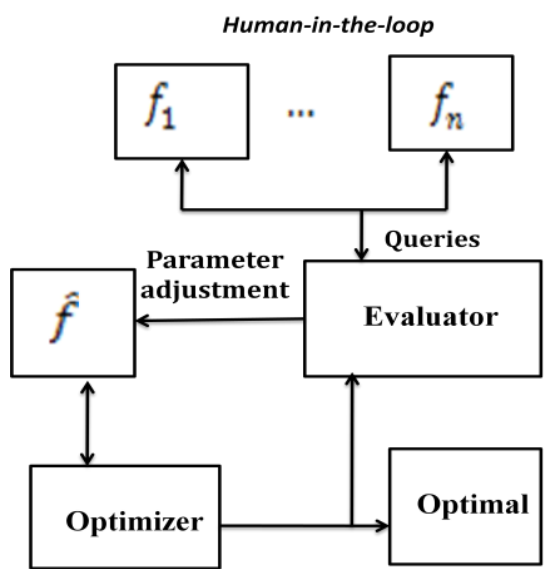

Fig 1: Schematic flowchart of the proposed optimization process [1]; learning the problem definition from the final user in interactive multiobjective optimization [4].

\section{CASE STUDY}

A reliable optimal design process for the Non-uniform rational B-spline (NURBS) curves and surfaces would have a wide and foundational application in CAGD, CAD, image processing, etc. Yet the optimal design and parameter tuning of the NURBS curves and surfaces is a complicated, highly non-linear and multiobjective optimization (MOO) problem.
The complexity of the problem is even increased when the criteria of product beauty is included to the design process. More on the problem, applications and previous approaches are available in [5], where the use of MOO algorithms enhances the design process by enabling optimization of several design objectives at once.

In this article the optimization process of NURBS including four conflicting and highly non-liner design objectives is described. For solving problems as such, with a high level of complexity, modeling the true nature of the problem is of importance and essential. For this reason a considerable amount of efforts is made in modeling the MOO problems in Scilab and the details are described.

\subsection{Statement of the case study}

A tensor product NURBS is defined as; $\quad S(u, v)=$ $\sum_{i=0}^{n-1} \sum_{j=0}^{m-1} \mathbf{P}_{\mathbf{i}, \mathbf{j}} R_{i, j}(s, t)$, where $\boldsymbol{P}_{\boldsymbol{i}, \boldsymbol{j}}$ are control points of the surface with the orders and the numbers of $n$ and $m . R_{i, j}(s, t)$ are the NURBS basis function, depended on the design variables including weights, $\boldsymbol{w}$, the knot vectors, $\boldsymbol{u} \& \boldsymbol{v}$, the $d_{u} \& d_{v}$ orders of the surface and the parameterization, $s \& t$.

Handling the parameterization, knot vectors and NURBS weights is described in [4]. Tuning NURBS weights and knot vector all together dramatically increases the number of DOF which is proportional to $n * m$.

According to the input points, $\boldsymbol{Q}_{i, j}$, and the design variables, the control points, $\boldsymbol{P}_{i, j}$, via utilizing the linear least squares fitting, are calculated and the surface is created [11].

Let $\boldsymbol{M}$ be the collocation matrix used for surface fitting; $\boldsymbol{Q}_{x}, \boldsymbol{Q}_{y}, \boldsymbol{Q}_{z}$ are the coordinates of $Q$, the data to be fitted; $\operatorname{diag}(x)$ a diagonal matrix whose entries are the vector $x$.

$$
\begin{array}{ccc}
t=M * w \quad X & =\operatorname{diag}\left(Q_{x}\right) \quad Y=\operatorname{diag}\left(Q_{y}\right) & Z \\
& =\operatorname{diag}\left(Q_{z}\right) & \\
& v_{x}=X * t & v_{y}=Y * t \quad v_{z} \\
& =Z * t &
\end{array}
$$

The position of the surface's control points $\boldsymbol{p}_{x}, \boldsymbol{p}_{y}, \boldsymbol{p}_{z}$ are given by least solution of the following equations: $d_{x}=M *$ $v_{x}, d_{y}=M * v_{y}, \quad d_{z}=M * v_{z}$

\subsection{Optimization Objectives}

The goal of the optimization process is to produce a set of NURBS surfaces which approximates a set of input points, $\boldsymbol{Q}$ $=\boldsymbol{Q}_{0,0}, \ldots, \boldsymbol{Q}_{N-1, M-1} \in \mathbb{R}^{d}, d=2,3$, and are optimal with respect to the specified design objectives. Once the surface is created the quality of it could be considered by evaluating a set of specified design objectives, i.e. $O_{1}(S(s, t))$, . . ., $O_{k}(S(s, t)$.

Approximation Error, $O_{1}$, the distance between the surface and the points $\boldsymbol{Q}$ measured at the parametrization points $\boldsymbol{s}_{\boldsymbol{i}}, \boldsymbol{t}_{\boldsymbol{i}}$, is often subjected to minimization

$$
\begin{aligned}
& O_{1}=\min \left(\sum_{i=0}^{n-1} \sum_{j=0}^{m-1}\left\|\boldsymbol{S}\left(s_{i}, t_{i}\right)-\boldsymbol{Q}_{i, j}\right\|^{2}\right), \text { under } L_{2} \text { norm, } \\
& O_{1}=\max \left(\left\|\boldsymbol{S}\left(s_{i}, t_{i}\right)-\boldsymbol{Q}_{i, j}\right\|\right), i=0, \ldots, n-1 ; j= \\
& 0, \ldots, m-1, \text { under } L_{\infty} \text { norm. }
\end{aligned}
$$

Surface Area, $\mathrm{O}_{2}$, in conflict with approximation error, controls artifacts due to over-fitting; $\quad O_{2}=\int_{0}^{1} \int_{0}^{1} \| \frac{d s}{d s} \times$ $\frac{d s}{d t} \| d s d t$. 
Surface Elastic Energy, $\mathrm{O}_{3}$, as an other conflicting objective is a highly non-linear term;

$O_{3}=\int_{0}^{1} \int_{0}^{1}\left\|k_{\min }^{2}+k_{\text {max }}^{2}\right\| d A$, where $A$ is the surface area.

\section{THE PROPOSED AND FORMER APPROACHES}

The mathematical modeling of the NURBS curves and surfaces design problem results in a multiobjective optimization problem which cannot be handled as such by traditional single objective optimization algorithms. Considering the problem with Conjugate Gradient and Newton based approaches, the optimization process is divided into several phases and each functional is optimized separately [7, 8, 9]. In this approach the multiobjective problem is solved via a single objective optimization algorithm. However the results obtained clearly are not promising.

Previously an evolutionary MOO algorithm [5] is used to handle this case. In this approach the results are reported promising due to the robustness and efficiency of evolutionary algorithms. Evolutionary algorithms [9] are natural choice for multiobjective optimization since at each step the algorithms keeps a population, which is a set of solutions instead of a single, optimal, solution. Because of the robustness and efficient handling of highly non-linear objective functions and constrains the use of EA in geometrical problem has proved to be a powerful technique [11]

In the proposed brain-computer algorithms, in contrast to the evolutionary algorithms, the DM guides the optimization in the desirable search locations and the final desirable surface. In this case the computation cost is minimized and the preferences of the decision maker are effectively considered.

Here, as an alternative to the previous approaches the robust and interactive MOO algorithm of RSO [1,4] is proposed in order to efficiently optimize all the design objectives at once including the criteria of beauty in which couldn't be completely considered in the previous attempts. In this framework the quality of the surface, similar to the previous research workflows, is measured using a set of certain functions, then an optimization algorithm is applied in order to optimize the function to improve the quality of the surface.

The problem is modeled in scilab and the model is integrated to the optimizer via advanced interfaces to the RSO algorithm and its brain-computer evolutionary multiobjective optimization implementations and visualization $[1,13]$. In this framework the application of learning and intelligent optimization and reactive business intelligence approaches in improving the process of such complex optimization problems are described. Furthermore the problem is further reconsidered by reducing the dimensionality and the dataset size [12], multi-dimensional scaling, clustering and visualization tools [21-28].

\section{CONCLUTIONS}

A brain-computer approach characterized by its ability to learn an arbitrary utility function from a DM expressesing preferences between couples of selected solutions. The method is based on Support Vector Machines and derived tools from the machine learning community. The optimization methodology of Reactive Optimization based on the paradigm of learning while optimizing is utilized. The presented study case demonstrates the feasibility and effectiveness of the brain-computer algorithm with both linear and non-linear user preferences.

\section{ACKNOWLEDGMENTS}

The author would like to thank Estonian Institute for funding this research with the grant of Estophilus which is provided under the programme of academic studies of Estonian language and culture abroad.

\section{REFERENCES}

[1] Bowman, R. Battiti and P. Campigotto, Reactive search optimization: Learning while optimizing. an experiment in interactive multi-objective optimization. In S. Voss and M. Caserta, editors, Proceedings of MIC 2009, VIII Metaheuristic International Conference, Lecture Notes in Computer Science. Springer Verlag, 2010.

[2] A. Mosavi, On Engineering Optimization the Splined Profiles, in Proceedings of International modeFRONTIER Users' Meeting, Trieste, Italy, 2010.

[3] A. Mosavi, "Multiobjective Optimization of Spline Curves Using Mode Frontier," Proceedings of International Conference on Engineering Optimization and International Mode Frontierusers' Meeting, Trieste, 2010.

[4] R. Battiti, M. Brunato, Reactive Business Intelligence From Data to Models to Reactive Search Srl, Italy, February 2011.

[5] R. Goldenthal, M. Bercovier, Design of Curves and Surfaces by Multiobjective Optimization, Mathematical Methods for Curves and Surfaces, 2004.

[6] B. Roberto, A. Passerini "Brain-Computer Evolutionary Multi-Objective Optimization (BC-EMO): a genetic algorithm adapting to the decision maker." (PDF). IEEE Transactions on Evolutionary Computation 14 (15): 671-687, 2010.

[7] M.I.G. Bloor, M.J. Wilson, H. Hagen, The smoothing properties of variational schemes for surface design, Computer Aided Geometric Design,Vol. 12, pp. 381394, 1995.

[8] G. Brunnett, H. Hagen, P. Santarelli, Variational design of curve and surfaces, Surveys on Mathematics for Industry, Vol 3. pp. 1-27, 1993.

[9] G. Brunnett, J. Kiefer, Interpolation with minimalenergy splines, Computer-Aided Design, Vol. 26, No. 2, pp. 137-144, 1994.

[10] K. Deb, , Evolutionary algorithms for multi-criterion optimization in engineering design, Evolutionary Algorithms in Engineering and Computer Science, Miettinen K., Makela, M. M., Neittaanmaki, P., and Periaux J. (eds.), John Wiley Sons, Ltd, Chichester, UK, pp. 135-161, 1999.

[11] G. Renner, (ed.), Computer-Aided Design, Genetic Algorithms, Vol 35, Issue 8. pp. 707-769, 2003.

[12] A. Mosavi, Multiple criteria decision-making preprocessing using data mining tools. IJCSI International Journal of Computer Science Issues, V7, Issue 2, No 1, 2010.

[13] J. Branke, K. Deb, K. Miettinen, and R. S lowinski, Eds., Multiobjective Op- timization: Interactive and 
Evolutionary Approaches. Berlin, Heidelberg: SpringerVerlag, 2008.

[14] K. Miettinen, F. Ruiz, and A. Wierzbicki, Introduction to Multiobjective Optimization: Interactive Approaches," in Multiobjective Optimization: Interactive and Evolutionary Approaches. SpringerVerlag Berlin, Heidelberg, 2008, pp. 27-57.

[15] J. D. Schaer, Multiple objective optimization with vector evaluated genetic algorithms," in Proceedings of the 1st International Conference on Genetic Algorithms. Hillsdale, NJ, USA: L. Erlbaum Associates Inc., 1985, pp. 93-100.

[16] K. Deb, Multi-objective optimization using evolutionary algorithms. Wiley, 2001.

[17] F. di Pierro, K. Soon-Thiam, and D. Savic, \An investigation on preference order ranking scheme for multiobjective evolutionary optimization," Evolutionary Computation, IEEE Transactions on, vol. 11, no. 1, pp. 17- 45, Feb. 2007.

[18] I. Das, A preference ordering among various pareto optimal alternatives," Structural and Multidisciplinary Optimization, vol. 18, no. 1, pp. 30\{35, 1999.

[19] J. March, Bounded rationality, ambiguity, and the engineering of choice, The Bell Journal of Economics, pp. $587\{608,1978$.

[20] A. Mosavi and A. Vaezipour, "Reactive Search Optimization; Application to Multiobjective Optimization Problems," Applied Mathematics, Vol. 3 No. 10A, 2012, pp. 1572-1582.

[21] A. Mosavi, M. Hoffmann and A. S. Milani, "Optimal Design of the NURBS Curves and Surfaces Utilizing
Multiobjective Optimization and Decision Making Algorithms of RSO," Conference of PhD Students in Mathematics, Szeged, Jnue 2012.

[22] A. Mosavi, M. Hoffmann and A. S. Milani, "Adapting the Reactive Search Optimization and Visualization Algorithms for Multiobjective Optimization Problems; Application to Geometry," Conference of PhD Students in Computer Science, Szeged, June 2012.

[23] A. Mosavi, Engineering Design and Decision-Making Models, Doctoral Dissertation, University of Debrecen, 2013.

[24] A. Mosavi, A multicriteria decision making environment for engineering design and production decision-making. International Journal of Computer Applications, 69(1), 26-38, 2013.

[25] A. Mosavi, On developing a decision-making tool for general applications to computer vision. International Journal of Computer Applications, Special Issue on Recent Trends in Pattern Recognition and Image Analysis 1: 10-17, 2013.

[26] A. Mosavi, Optimal Engineering Design. Tech. Rep. 2013. University of Debrecen, Hungary, 2013.

[27] A. Mosavi. Decision-making Software Architecture; the Visualization and Data Mining Assisted Approach. International Journal of Information and Computer Science, 3 (1), 12-26, 2014.

[28] A. Mosavi, Decision-Making Models for Optimal Engineering Design and their Applications, 2013. 